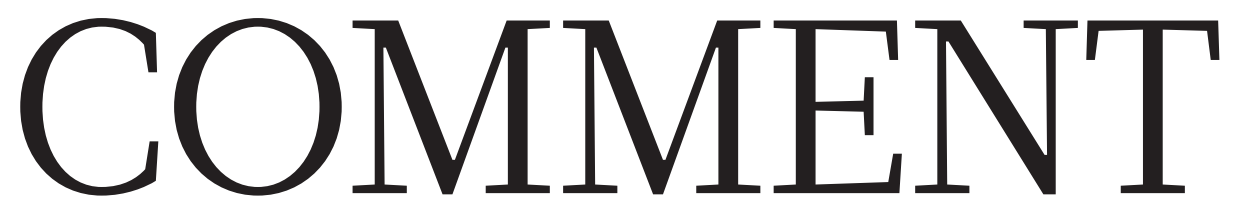

DISASTERS Climate, fires and floods are linked - study them together $\mathbf{p . 4 5 8}$

\section{GENOMES Don't use \\ Blupprint genomics to excuse \\ Social inequality $\mathbf{p . 4 6 1}$}

AUTHORSHIP Follow the

film industry and list

contributions instead $\mathbf{p . 4 6 4}$
CONFERENCES Do boring

speakers talk for longer, or does it just feel that way? p.464

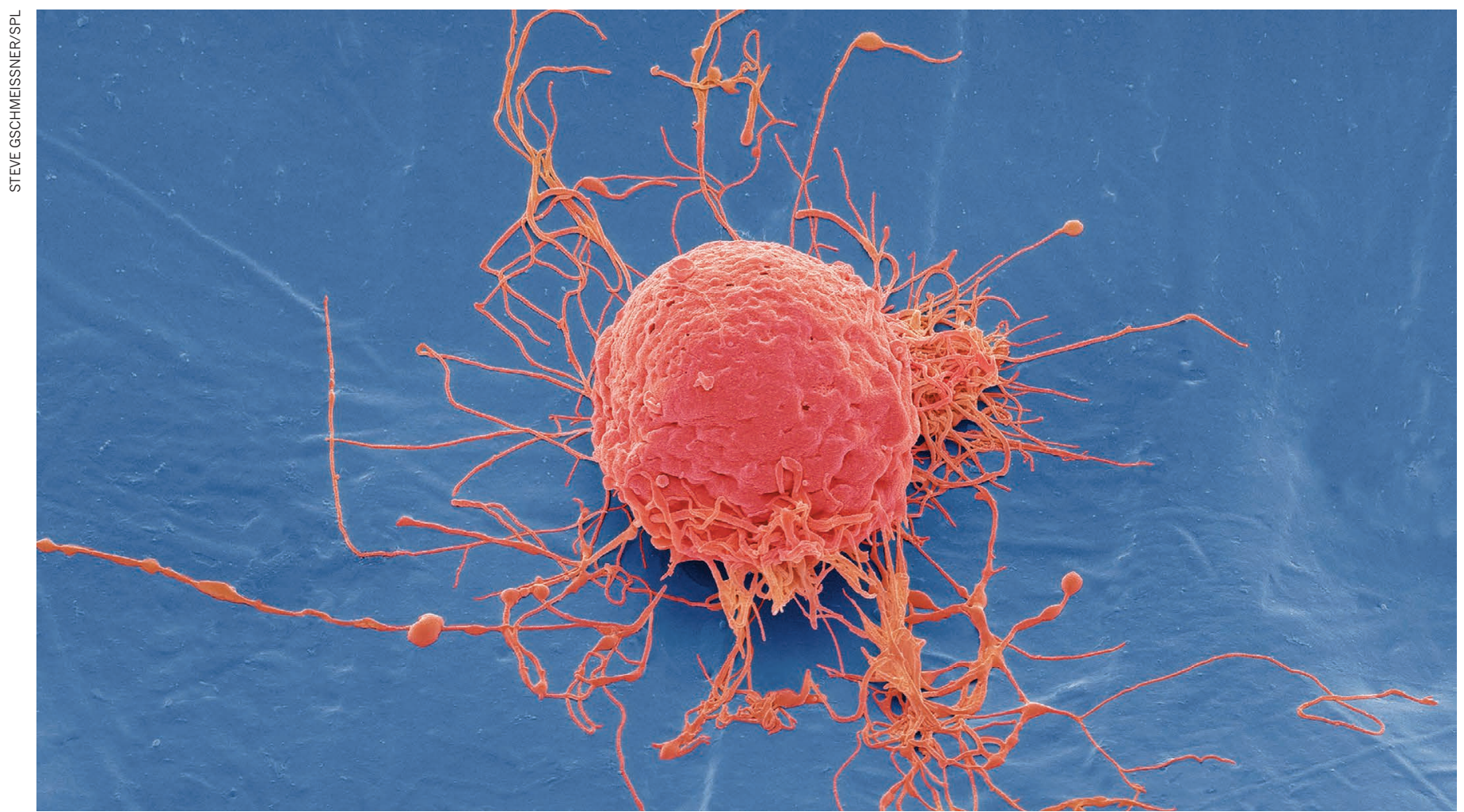

Scanning electron micrograph of what is called a human mesenchymal stem cell, which some say can develop into bone, cartilage or fat cells.

\title{
Clear up this stem-cell mess
}

\section{Confusion about mesenchymal stem cells is making it easier for people to sell unproven treatments, warn Douglas Sipp, Pamela G. Robey and Leigh Turner.}

$\mathrm{V}$

arious populations of cells in the adult human body have been the subject of controversy since the early 2000s. Contradictory findings about these haphazardly termed 'mesenchymal stem cells', including their origins, developmental potential, biological functions and possible therapeutic uses, have prompted biologists, clinicians and scientific societies to recommend that the term be revised or abandoned. Last year, even the author of the paper that first used the term mesenchymal stem cells (MSCs) called for a name change ${ }^{1}$.

Tissue-specific stem cells, which have a limited ability to turn into other cell types, are the norm in most of the adult body.
Several studies indicate that the variety of cells currently dropped into the MSC bucket will turn out to be various tissuespecific cell types, including stem cells ${ }^{2}$.

Yet the name persists despite the evidence pointing to this, and almost two decades after questions about the validity of MSCs were first raised. A literature search indicates that, over the past 5 years, more than 3,000 research articles referring to MSCs have been published every year (see 'Tenacious term'). And several national regulatory agencies have now licensed MSC-based drugs, although most of these approvals have been provisional or are based on underpowered studies (see 'Doubtful drugs').

In our view, the wildly varying reports have helped MSCs to acquire a near-magical, all-things-to-all-people quality in the media and in the public mind ${ }^{3}-$ hype that has been easy to exploit. MSCs have become the go-to cell type for many unproven stemcell interventions. The confusion must be cleared up.

What is needed is a coordinated global effort to improve understanding of the biology of the cells currently termed MSCs, and a commitment from researchers, journal editors and others to use more-precise labels. We must develop standardized analyses of gene expression, including on a cell-by-cell basis, and rigorous assays to establish the precise products of cell differentiation in various tissues. Such $>$ 
$>$ efforts could put an end to lingering questions about MSC identity and function, once and for all.

\section{A CONTROVERSIAL CELL}

The MSC concept dates to a 1991 paper $^{4}$ in which US biologist Arnold Caplan described the isolation of a type of stem cell found in bone marrow, building on reports by other groups (see, for example, ref. 5). Collectively, this work showed that certain cells in the supportive tissue of bone marrow (the stroma) could differentiate into cartilage, bone and fat, and provide some of the signals needed for haematopoietic stem cells (the immature precursors of all blood cells) to give rise to blood-cell types.

Over the next decade, there was an explosion in the number of tissue types in which MSCs were reported. And there was a similar jump in the diversity of cell types that MSCs were reportedly able to differentiate into. Yet by the early 2000s, it had become clear that separate labs were using different cell-surface markers to characterize MSCs. In 2006, a working group of the International Society for Cellular Therapy (ISCT) acknowledged the "inconsistencies and ambiguities" and recommended a new designation: multipotent mesenchymal stromal cells ${ }^{6}$.

Despite the ISCT's recommendation, the stem-cell designation has proved strangely durable. Assays commonly used to evaluate a cell's 'stemness' are prone to misinterpretation. Many researchers have failed to take into account the developmental origins of the tissues they cite as sources of MSCs, or to observe the rigorous definition of a stem cell. Also, the very commonness of the MSC term seems to have consolidated its acceptance.

In the past few years, however, there has been further questioning of the MSC as a valid biological entity. A 2014 study $^{7}$ by researchers at the US Food and Drug Administration found almost no agreement in the molecular characterization of MSCs among the investigational new drug applications submitted to the agency. A 2016 study ${ }^{2}$ likewise found that various cell populations from different tissues, all classed as MSCs, actually differ drastically in their gene expression and in their ability to differentiate. And last year, Caplan revealed that he no longer believes that MSCs are stem cells ${ }^{1}$.

In that article, Caplan implores the scientific community to adopt yet another moniker: medicinal signalling cells. (According to Caplan, medicinal signalling cells, identified on the basis of cell-surface proteins and their ability to turn into other cell types in vitro, home in on sites of injury. There they secrete cocktails of proteins that modulate the immune response, reduce inflammation, promote wound healing and inhibit cell death.)

Since 1991, more than 32,000 Medlineindexed articles referring to "mesenchymal stem cells" have been published. Our reading of a subset of these - many hundreds over the past decade - suggests that the field continues to be a mess.

According to the literature, MSCs are most often isolated from bone marrow and adipose tissue (fat). They have also been identified in perinatal tissues - umbilical cord, Wharton's jelly (a gelatinous substance found in the umbilical cord), the amnion (the membrane surrounding the embryo) and the placenta - as well as in other sources, including baby teeth and menstrual blood. Some groups report that MSCs are most common in fat. Others state that they are associated with blood vessels throughout the body's connective tissues. Some note that MSCs are exceedingly rare; others say they are abundant.

Canonically, MSCs (including those from non-skeletal sources) are supposed to give rise to cartilage, bone and fat. But reports exist of their differentiation into muscle, endothelium and various cells of the heart, liver and kidney, as well as of the nervous and reproductive systems. Some researchers and clinical providers have even described MSCs as nearly pluripotent, meaning that they can differentiate into almost every cell type in the adult body. Moreover, there are countless claims of therapeutic uses for these cells in multiple unrelated diseases, ranging from arthritis and diabetes to Parkinson's disease and autism.

\section{SALES PITCH}

All of the disagreement in the scientific community about the nature of MSCs, and even about their existence, is almost certainly making it easier for businesses to sell treatments allegedly based on MSCs.

Direct-to-consumer marketing of unapproved stem-cell treatments for medical conditions has exploded in the past five years, particularly in the United States, Australia and Japan. A 2016 report $^{8}$ documented $^{2}$ 351 US companies selling putative stem-cell treatments direct to consumers; almost half refer to MSCs in their marketing materials.

Businesses have been quick to capitalize on the conflicting claims in the literature ${ }^{9}$. To convey that MSCs can treat a wide range of diseases and injuries, firms selectively highlight those articles suggesting that MSCs are easy to harvest and can give rise to other cell types, or those indicating that the cells secrete all sorts of healing factors. (The large body of research indicating that tissue-specific stem cells have more limited roles is overlooked.)

Interestingly, some publicly traded companies have shown increasing reluctance to define their products as mesenchymal stem cells. Cytori Therapeutics in San Diego, California, a developer of devices for harvesting and processing cells from patients, has begun to describe its target cell population as 'adipose-derived regenerative cells' instead of calling them MSCs.

\section{SOLUTIONS IN SCIENCE}

What can be done to clear up the confusion? In our view, re-categorizing MSCs as 'stromal' or 'signalling' cells won't help.

Some researchers are following the ISCT recommendations, but judging by the thousands of papers on MSCs still being published every year, many are not. Meanwhile, Caplan's proposal could introduce more problems than it solves. There is strong evidence for the existence of a tissue-specific stem cell in bone-marrow stroma at least, albeit one with a limited ability to differentiate into other cell types. Also, the use of 'medicinal' instead of 'mesenchymal' could encourage the assumption that MSCs have broad therapeutic usefulness before robust evidence for this has been obtained.

We think that answers will be found in better science. After all, many researchers now doubt that a single multipotent stem cell (meaning one that can give rise to several other kinds of cell) is present throughout the adult body, thanks to the rigour and persistence of some stem-cell biologists.

The reliable identification of tissue-specific cells - stem cells or otherwise - should involve omics approaches, such as those designed to analyse the gene-expression patterns of a cell or its protein content, and rigorous assays to establish what a particular cell can differentiate into, either in vivo

\section{DOUBTFUL DRUGS}

\section{Clinical trials with MSCs fail to deliver}

A move towards translational studies requires a robust understanding of the actual biological properties of the cell types currently called mesenchymal stem cells (MSCs).

So far, both the results and the quality of MSC-based clinical trials have been underwhelming. Take a 2014 meta-analysis of 49 trials using 'bone-marrow stem cells' (in many cases, 'bone-marrow MSCs') to treat cardiovascular disease, for instance.
According to that analysis, the studies that scored better in terms of rigour were more likely to report less efficacy for MSC treatments than were those judged to be less rigorous ${ }^{11}$.

Clinical studies using MSCs (or any stem cells) must adhere to the same standards of research design and oversight that apply to any responsible clinical trial before the cells are administered to human participants. D.S., P.G.R. \& L.T. 


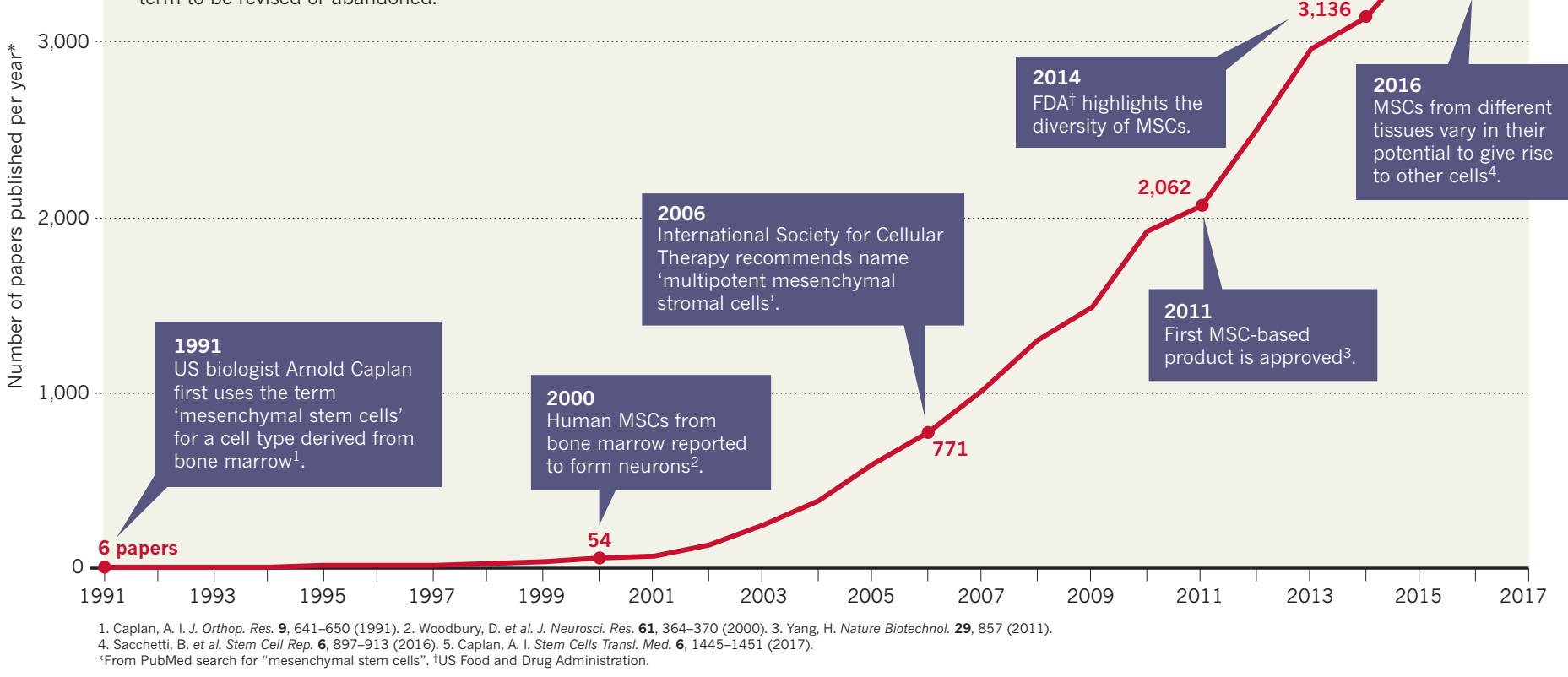

or in vitro. (Historically, inconsistencies in surface markers and stains, or the use of unreliable morphological characteristics, have led to frequent errors when identifying cell types ${ }^{10}$.)

Ongoing initiatives such as the Human Cell Atlas, an effort to characterize all cells in the body, could shed light on the cellular components of specific tissues. But, ultimately, resolving the MSC identity crisis is likely to require a focused undertaking by stem-cell biologists - similar to a series of projects conducted in the 2000 s by the International Stem Cell Forum to characterize pluripotent stem cells. (The forum is a collaboration designed to support stem-cell research.)

As part of the push for better science, regulatory agencies and editors of influential stem-cell and general scientific journals will need to develop and enforce rigorous methodological standards.

\section{DISPEL THE MSC MYTH}

Meanwhile, the community needs to stop subsuming multiple cell types under one catch-all phrase.

Scientific societies, such as the International Society for Stem Cell Research (ISSCR) and national stem-cell organizations, should consider whether MSC studies ought to be presented at stem-cell conferences under the MSC umbrella. Clinical-trial registries, such as clinicaltrials.gov, should also exercise heightened scrutiny over studies in which MSCs are described as the investigational product. (Hundreds of such studies are already listed on international clinicalresearch databases.) Groups studying tissuespecific stem cells should be encouraged to

rethink their choice of terms. Both bone marrow and fat, for example, contain stemcell populations that help to maintain these tissues. And referring to these as skeletal or adipose stem cells could help to vanquish the myth of a near-ubiquitous, all-powerful adult MSC $^{2}$. Certainly, studies of poorly characterized cellular miscellanies should not be accepted without rigorous evidence of multipotency and self-renewal.

Journal editors, editorial boards and reviewers should similarly be more exacting when accepting or approving MSC

"In most cases,
rigorous
preclinical
studies of
these cells
are limited or
non-existent."
reports for publication in journals. And funding bodies must consider whether studies using MSCs qualify for funds earmarked for stem-cell research.

Journalists can also play an important part in combating MSC misconceptions. When reporters write stories about clinics selling 'mesenchymal stem-cell treatments' and other purported stem-cell therapies, they should inform their audiences that scientists debate whether such cells are, in fact, stem cells. They should also make it clear that, in most cases, rigorous preclinical studies of these cells are limited or non-existent.

Meanwhile, organizations such as the ISSCR, the ISCT and national stem-cell societies should ensure that guidebooks, educational videos and other communication tools designed for people receiving 'adult stem-cell therapies' are updated to reflect the demise of the MSC as a viable concept.
Whatever the ultimate identity and biological activity of the cells formerly known as MSCs, the scientific principles that guide their clinical development and use must be the same as those for other new therapies: precision, validation, characterization, objectivity and the abandonment of terms and conceptual models that mislead more than they illuminate.

Douglas Sipp is a researcher at RIKEN and project professor at Keio University School of Medicine and Global Research Institute, Tokyo, Japan. Pamela G. Robey is a senior investigator at the National Institute of Dental and Craniofacial Research, National Institutes of Health, Department of Health and Human Services, Bethesda, Maryland, USA. Leigh Turner is associate professor at the Centre for Bioethics, University of Minnesota, Minneapolis, USA. e-mail:sipp@cdb.riken.jp

1. Caplan, A. I. Stem Cells Transl. Med. 6, 1445-1451 (2017).

2. Sacchetti, B. et al. Stem Cell Rep. 6, 897-913 (2016).

3. Caulfield, T., Sipp, D., Murry, C. E., Daley, G. Q. \& Kimmelman, J. Science 352, 776-777 (2016).

4. Caplan, A. I. J. Orthop. Res. 9, 641-650 (1991).

5. Owen, M. \& Friedenstein, A. J. Ciba Found. Symp. 136, 42-60 (1988).

6. Dominici, M. et al. Cytotherapy 8, 315-317 (2006).

7. Mendicino, M., Bailey, A. M., Wonnacott, K., Puri, R. K. \& Bauer, S. R. Cell Stem Cell 14, 141-145 (2014).

8. Turner, L. \& Knoepfler, P. Cell Stem Cell 19, 154-157 (2016).

9. Sipp, D. et al. Sci. Transl. Med. 9, eaag0426 (2017).

10.Bianco, P., Robey, P. G. \& Simmons, P. J. Cell Stem Cell 2, 313-319 (2008).

11. Nowbar, A. N. et al. Br. Med. J. 348, g2688 (2014).

L.T. declares competing non-financial interests; see go.nature.com/2pjhdai for details. 